\title{
Quantitative MRI reveals differences in striatal myelin in children with DLD
}

\author{
Saloni Krishnan ${ }^{1,2}$ \\ Gabriel J. Cler ${ }^{1,3}$ \\ Harriet J. Smith ${ }^{1,4}$ \\ Hanna E. Willis ${ }^{1,5}$ \\ Salomi S. Asaridou ${ }^{1,6}$ \\ Máiréad P. Healy ${ }^{1,7}$ \\ Daniel Papp ${ }^{8}$ \\ Kate E. Watkins ${ }^{1}$
}

${ }^{1}$ Wellcome Centre for Integrative Neuroimaging, Dept of Experimental Psychology,

University of Oxford, OX2 6GG, Oxford, UK

${ }^{2}$ Department of Psychology, Royal Holloway, University of London, Egham Hill, Surrey TW20 OEX

${ }^{3}$ Department of Speech and Hearing Sciences, University of Washington, Seattle WA

${ }^{4}$ MRC Cognition and Brain Sciences Unit, University of Cambridge, 15 Chaucer Road, Cambridge CB2 7EF, UK

${ }^{5}$ Nuffield Department of Clinical Neurosciences, John Radcliffe Hospital, Headley Way, Headington, Oxford OX3 9DU

${ }^{6}$ Department of Experimental Psychology, University College London, 26 Bedford Way, London WC1H OAP

${ }^{7}$ Department of Psychology, University of Cambridge, Downing Street, Cambridge CB2 3EB

${ }^{8}$ Wellcome Centre for Integrative Neuroimaging, FMRIB Centre, Nuffield Department of Clinical Neuroscience, University of Oxford, OX3 9DU, Oxford, UK

\section{Corresponding author:}

Dr. Saloni Krishnan

Department of Psychology, Royal Holloway, University of London, Egham Hill, Surrey TW20 $O E X$

<saloni.krishnan@rhul.ac.uk> 


\title{
Quantitative MRI reveals differences in striatal myelin in children with DLD
}

\begin{abstract}
Developmental Language Disorder (DLD) is a common neurodevelopmental disorder characterised by receptive or expressive language difficulties or both. While theoretical frameworks and empirical studies support the idea that there may be neural correlates of DLD in frontostriatal loops, findings are inconsistent across studies. Here, we use a novel semiquantitative imaging protocol - multiparameter mapping (MPM) - to investigate microstructural neural differences in children with DLD. The MPM protocol allows us to reproducibly map specific indices of tissue microstructure. In 56 typically developing children and 34 children with DLD, we derived maps of: 1) longitudinal relaxation rate R1 (1/T1); 2) the transverse relaxation rate R2* $(1 / \mathrm{T} 2 *)$; and 3) Magnetization Transfer Saturation (MTsat). $\mathrm{R} 1$ and MTsat predominantly index myelin, while R2* is sensitive to iron content. Children with DLD showed reductions in MTsat values in the caudate nucleus bilaterally, as well as in the left ventral sensorimotor cortex and Heschl's gyrus. They also had globally lower R1 values. No group differences were noted in R2* maps. Differences in MTsat and R1 were coincident in the caudate nucleus bilaterally. These findings support our hypothesis of corticostriatal abnormalities in DLD and indicate abnormal levels of myelin in the dorsal striatum in children with DLD.
\end{abstract}




\section{Quantitative MRI reveals differences in subcortical microstructure in children with DLD}

Children with Developmental Language Disorder (DLD) struggle to learn their native language for no obvious reason. DLD is an extremely common neurodevelopmental disorder, with recent estimates indicating the prevalence of DLD is 7\% (Norbury et al., 2016). DLD has serious economic and social consequences - it is associated with a higher risk for academic underachievement, unemployment, social and behavioural difficulties, and detriment to wellbeing (Conti-Ramsden et al., 2018). Although we know that DLD does not result from gross neural lesions, we still do not have a clear picture of how brain anatomy differs in children with DLD (Krishnan et al., 2016; Mayes et al., 2015). This is not only practically relevant but would also help us to understand the neural underpinnings of language development. Here, we use a robust new semiquantitative imaging protocol - MPM or Multi-Parameter Mapping (Weiskopf et al., 2013, 2021) - to shed light on microstructural neural differences in a large group of children with DLD.

There is a dearth of literature examining brain structure in children with DLD, which is surprising given the prevalence and impact of DLD. In the available literature, grey matter changes have been noted in language-relevant cortex, such as in the left inferior frontal gyrus and the posterior superior temporal gyrus (Badcock et al., 2012; Gauger et al., 1997; Jancke et al., 2007; Lee et al., 2020; Plante, 1991; Preis et al., 1998). Differences have also been noted in areas homologous to these language regions, such as the right perisylvian cortex (GirbauMassana et al., 2014; Jancke et al., 2007; Kurth et al., 2018). However, there is variability in the direction of differences reported in different studies - for instance, both increases and decreases in grey matter have been noted in the left inferior frontal gyrus (Badcock et al., 2012; Gauger et al., 1997; reviewed in Mayes et al., 2015). In addition to these cortical changes in the language network, we, and others, have hypothesised that the dorsal striatum is important for language learning, and may be abnormal in DLD (Krishnan et al., 2016; Ullman et al., 2020; Ullman \& Pierpont, 2005). The dorsal striatum is important for habitual and sequential learning (Graybiel \& Grafton, 2015; Yin \& Knowlton, 2006), and we hypothesise that it may play an important role in the acquisition of language because of the complexity of sequencing required for language. Indeed, in studies that probe complex sequential production in the vocal domain, the dorsal striatum is implicated (Rauschecker et al., 2008; Simmonds et al., 2014; Skipper et al., 2020). Additionally, a series of behavioural studies has suggested that sequential learning 
in the linguistic and non-linguistic domains is affected in children with DLD (Hsu \& Bishop, 2014; Lum et al., 2014; but see West et al., 2018, 2021). Previous studies of children with DLD analysing standard T1-weighted scans have also indicated reductions in the size of the caudate nucleus (Badcock et al., 2012; Herbert et al., 2003; Jernigan et al., 1991). Additionally, morphometric studies have also shown that affected members of the KE family (who have a point mutation in the FOXP2 gene and a behavioural profile similar to DLD) have reduced grey matter in the head of the caudate nucleus, areas within the sensorimotor cortex, the posterior inferior temporal cortex and the posterior lobe of the cerebellum, and increased grey matter in the putamen (Argyropoulos et al., 2019; Belton et al., 2003; Watkins et al., 2002). Other studies do not wholly support the view that the volume of the caudate nuclei is reduced in DLD but do indicate that there is some abnormality. While Lee and colleagues (2013) found reduced absolute volumes in the caudate nucleus and thalamus in individuals with DLD, these relationships did not survive correcting for total intracranial volume. The authors also observed negative relationships between language proficiency and the relative volume of subcortical structures such as the nucleus accumbens, globus pallidus, putamen and hippocampus. Similarly, Badcock et al. (2012) and Watkins et al. (2002) found negative relationships between nonword repetition and the volume of the right caudate nucleus. Others have suggested that volumetric differences in the caudate nuclei are modulated by age, with only younger children showing differences in volume (Soriano-Mas et al., 2009). Finally, some more recent studies suggest that children with DLD have greater grey matter in the right cerebellum (Pigdon et al., 2019). The interpretation of both cortical and subcortical findings is complicated by the heterogeneity of the DLD populations sampled, and the small sample sizes investigated. However, another factor that may also contribute to this lack of clarity is the nature of the scans acquired.

Standard structural imaging protocols such as T1-weighted scans reflect a complex mix of tissue properties, or in other words, the contrast between grey and white matter reflects a combination of histological properties such as iron content, myelin, cell density and water. Importantly, these microstructural properties yield regionally-specific contributions to commonly used structural markers such as grey matter volume or cortical thickness, which complicate the interpretation of these markers (Lorio et al., 2014, 2016). As standard T1weighted imaging protocols are dependent on acquisition parameters that can vary across scanners, they are also often difficult to replicate across studies. More recently, 
semiquantitative MRI methods have been used to map specific properties of tissue (Weiskopf et al., 2021). Semiquantitative protocols such as MPM can provide specific indices of microstructure, including myelin and macromolecular content of neural tissue, and the resulting maps are highly reproducible across individuals and scanners (Leutritz et al., 2020; Weiskopf et al., 2013). In the MPM quantitative imaging protocol, multiple maps are constructed, which allow us to probe different tissue properties. The generated maps quantify 1) the longitudinal relaxation rate $\mathrm{R} 1(1 / \mathrm{T} 1), 2)$ the transverse relaxation rate $\mathrm{R} 2 *(1 / \mathrm{T} 2 *)$, and 3) Magnetization Transfer Saturation (MTsat). The dominant influence on R1 in cortical tissue is myelin (Lutti et al., 2014), although R1 is sensitive to both myelin and iron in subcortical grey matter. R2* is sensitive to iron concentration, especially in ferritin-rich regions such as the striatum (Langkammer et al., 2010). MTsat is sensitive to bound water, and consequently myelin (Schmierer et al., 2004). This quantitative protocol therefore represents an unparalleled means of acquiring time-efficient, multi-modal, whole-brain data with insight into tissue composition. Such semiquantitative maps have been used to delineate heavily myelinated areas such as somatomotor (Carey et al., 2017), visual (Sereno et al., 2013), and auditory cortex (Dick et al., 2012). They have also been used to characterise developmental maturation in adolescence and young adulthood (Carey et al., 2018; NSPN Consortium et al., 2019; Paquola et al., 2019; Whitaker et al., 2016), during aging (Callaghan et al., 2014; Draganski et al., 2011; Steiger et al., 2016), and in pathological populations (Freund et al., 2013; Manara et al., 2019). A growing number of studies use these maps to understand brain-behaviour relationships (Allen et al., 2017; Clark et al., 2020). Most recently, we have used MPMs and found elevated iron levels in the putamen and speech motor network in people who stutter (Cler et al., 2021). However, this novel semiquantitative protocol has not yet been used to examine microstructure in children with neurodevelopmental disorders. A particular advantage of this protocol is that its sensitivity to cortical myelin can help distinguish two different explanations of developmental change in grey matter. Developmentally, cortical thinning indexed through grey/white matter contrast changes in standard T1-weighted scans could reflect a loss in the number of connections within grey matter, i.e., 'synaptic pruning' (Huttenlocher, 1979), or a gain in the volume of tissue through increased intra-cortical myelination that appears to "whiten" the grey matter on T1-weighted images (Paus, 2005). Recent studies using MPM protocols have shown that cortical thinning during development is associated with increased myelination rather than synaptic pruning (Natu et al., 2019; Whitaker et al., 2016). 
In the present study, we used the MPM quantitative imaging protocol to map contrast parameters (R1, R2* and MTsat) in typically developing children and those with DLD. We hypothesised that these indices would reveal that the microstructure of 1) the dorsal striatum (the caudate nuclei and the putamen) and 2) the left inferior frontal gyrus was altered in those with DLD.

\section{Results}

\section{Whole-brain comparisons of neural microstructure in children with DLD with typically developing (TD) children}

The two groups (TD and DLD) did not differ in in terms of age (see Supplementary Table 1, which also shows descriptive data summarising neuropsychological performance of the children included in these MPM analyses). There were no group differences in mean MTsat and R2* values for grey and white matter, or in total intracranial volume. The groups did differ in mean R1 values for grey matter (see Supplementary Table 2), in that children with DLD had lower R1 than the TD group across all grey matter. We created averages of MTsat, R1, and R2* for each group. Across all three maps, we observed high values in primary motor, visual, and auditory cortex (see Supplementary Figure 1), is line with our expectations. Additionally, we observed a close correspondence between average values of MTsat, R1, and R2* in grey matter and white matter in our two groups and published values (Weiskopf et al., 2013), see Supplementary Table 2.

We then investigated whether there were group differences in neural microstructure by assessing each of the parameter maps. Children with DLD had lower MTsat values than TD children in the left inferior frontal gyrus (pars opercularis), ventral sensorimotor cortex, insula cortex, lateral Heschl's gyrus, planum temporale, posterior superior temporal sulcus, and in portions of lateral and dorsomedial occipital cortex bilaterally. Subcortically, the children with DLD also had reduced MTsat in the dorsal caudate nucleus bilaterally; these differences were seen mainly in the body and were more extensive on the left than the right (Figure 1 and Supplementary Table 3). Consistent with the mean global differences in R1 in children with DLD (see Supplementary Table 2), examination of the R1 maps revealed widespread reduction over the lateral convexities of the frontal and parietal lobes bilaterally (but slightly more on the right), the medial frontal cortex including SMA and extending to paracentral lobule, and left 
bioRxiv preprint doi: https://doi.org/10.1101/2021.10.18.464793; this version posted October 20, 2021. The copyright holder for this preprint (which was not certified by peer review) is the author/funder, who has granted bioRxiv a license to display the preprint in perpetuity. It is made available under aCC-BY-NC 4.0 International license.

posterior temporal cortex extending from the superior temporal plane to posterior inferior temporal cortex. Subcortically, there were differences in R1 in the dorsal striatum and thalamus bilaterally, and in anterior portions of the medial temporal lobe (Figure 2). We did not find any significant group differences when examining the R2* maps. There were also no regions where children with DLD showed greater MT, R1 or R2* values relative to TD children.

Figure 1. $T D>D L D$ differences in MTsat values are shown on the surface. Axial slices show subcortical group differences in the caudate nuclei in red-yellow. The inset shows a boxplot of MTsat values in the caudate nuclei by group, with this cluster split into right and left using a hemispheric mask (red-DLD, blue-TD).

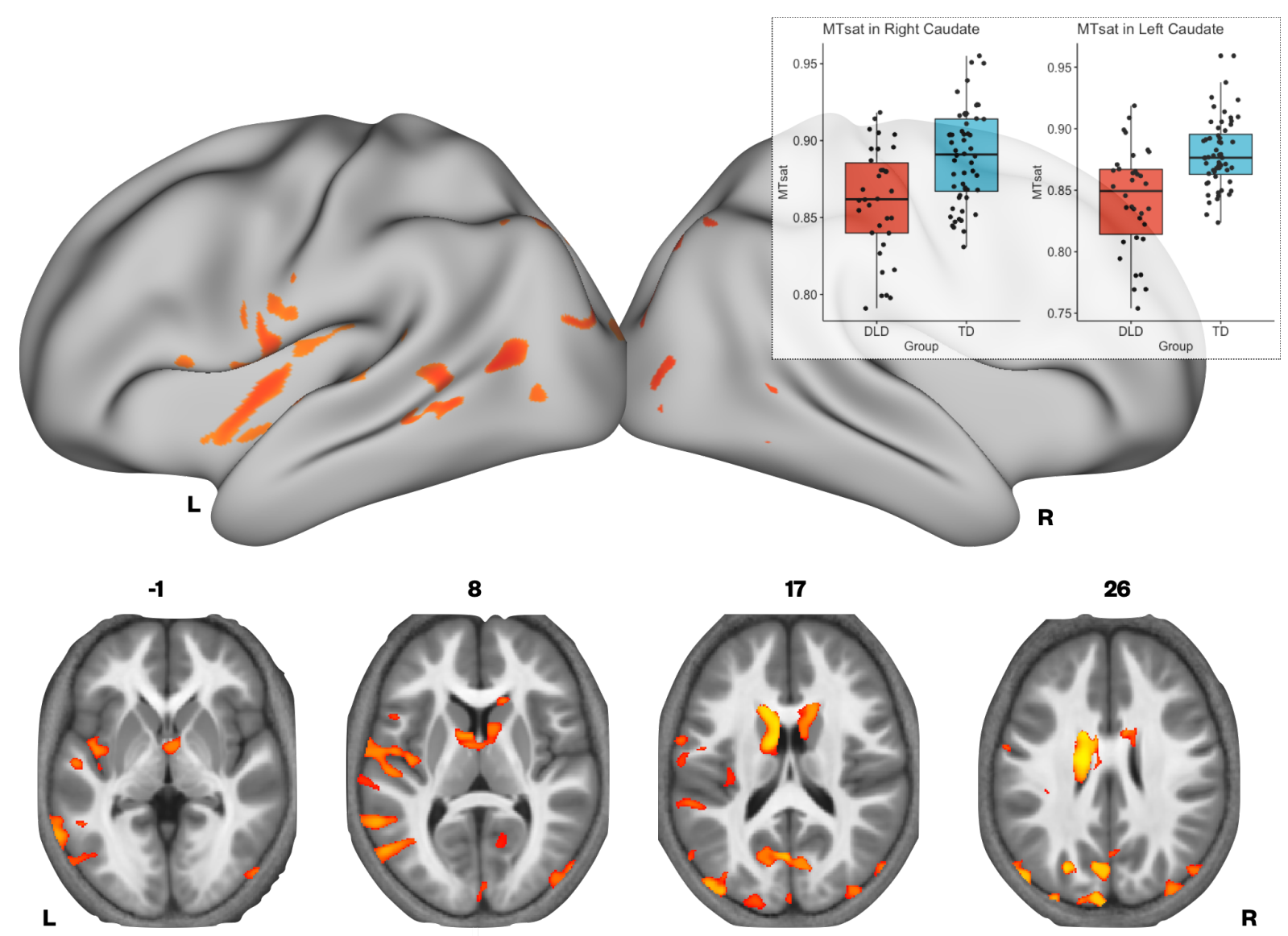


bioRxiv preprint doi: https://doi.org/10.1101/2021.10.18.464793; this version posted October 20, 2021. The copyright holder for this preprint (which was not certified by peer review) is the author/funder, who has granted bioRxiv a license to display the preprint in perpetuity. It is made available under aCC-BY-NC 4.0 International license.

Figure 2. TD $>$ DLD differences in R1 maps shown on the surface. Axial slices show subcortical group differences in the striatum in blue.

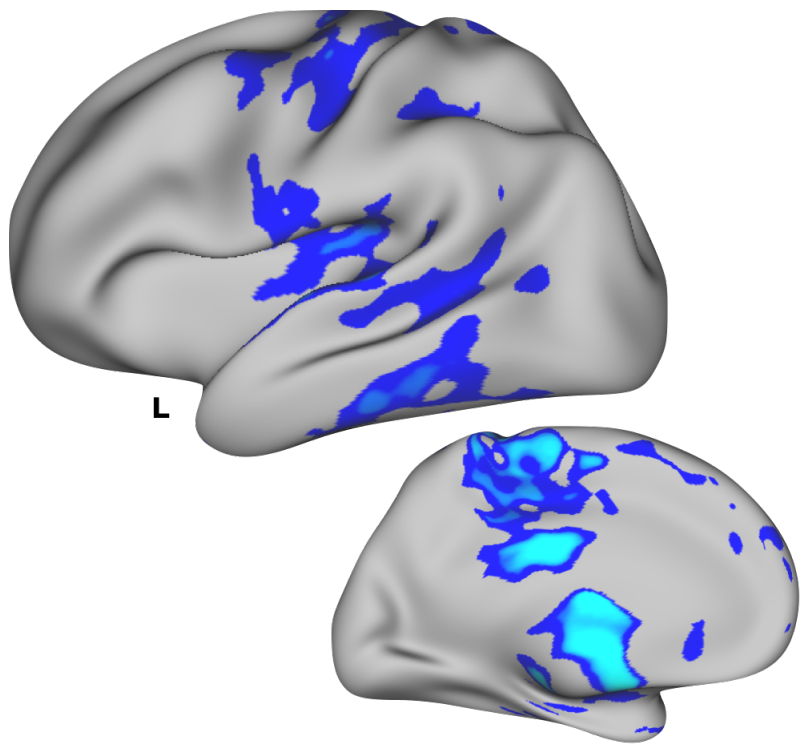

$-1$

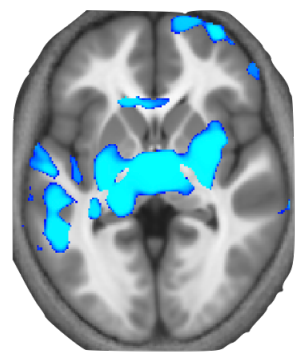

8

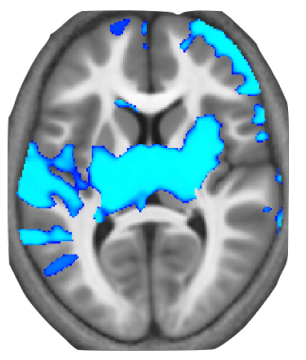

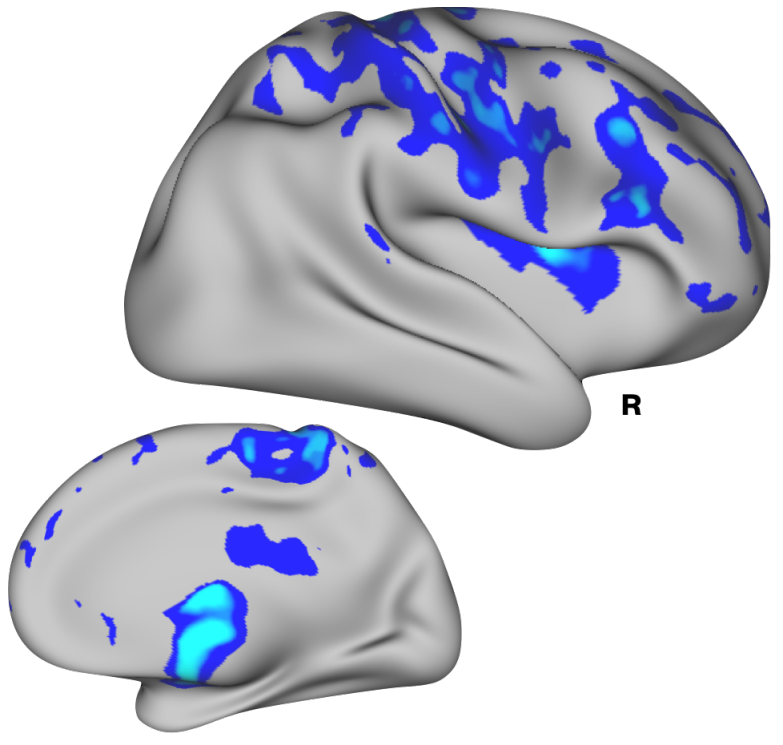

17

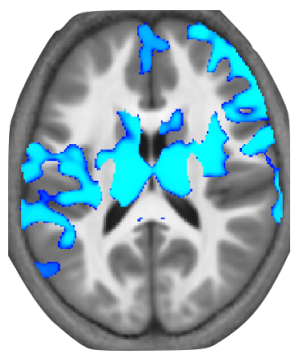

26

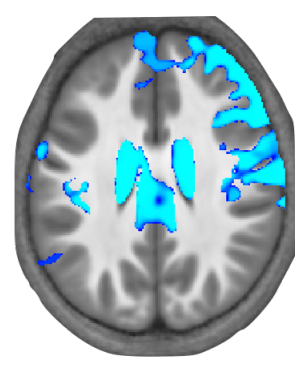

$\mathbf{R}$

Given that MTsat and R1 maps are particularly sensitive to myelin, and the R2* maps did not differ between the groups, we examined if there were regions where we would see convergence of differences across MTsat and R1. A conjunction analysis was performed, where we assessed which voxels showed significant TD $>$ DLD differences in both MTsat and R1 maps. We found conjoint differences in the MT and R1 maps across several brain regions, including the caudate nuclei bilaterally, and in the left ventral sensorimotor cortex, insula, lateral Heschl's gyrus, planum temporale, posterior superior temporal sulcus and middle temporal gyrus (Figure 3 and Supplementary Table 4). 
bioRxiv preprint doi: https://doi.org/10.1101/2021.10.18.464793; this version posted October 20, 2021. The copyright holder for this preprint (which was not certified by peer review) is the author/funder, who has granted bioRxiv a license to display the preprint in perpetuity. It is made available under aCC-BY-NC 4.0 International license.

Figure 3. Convergence of $T D>D L D$ differences in MTsat and $R 1$ maps
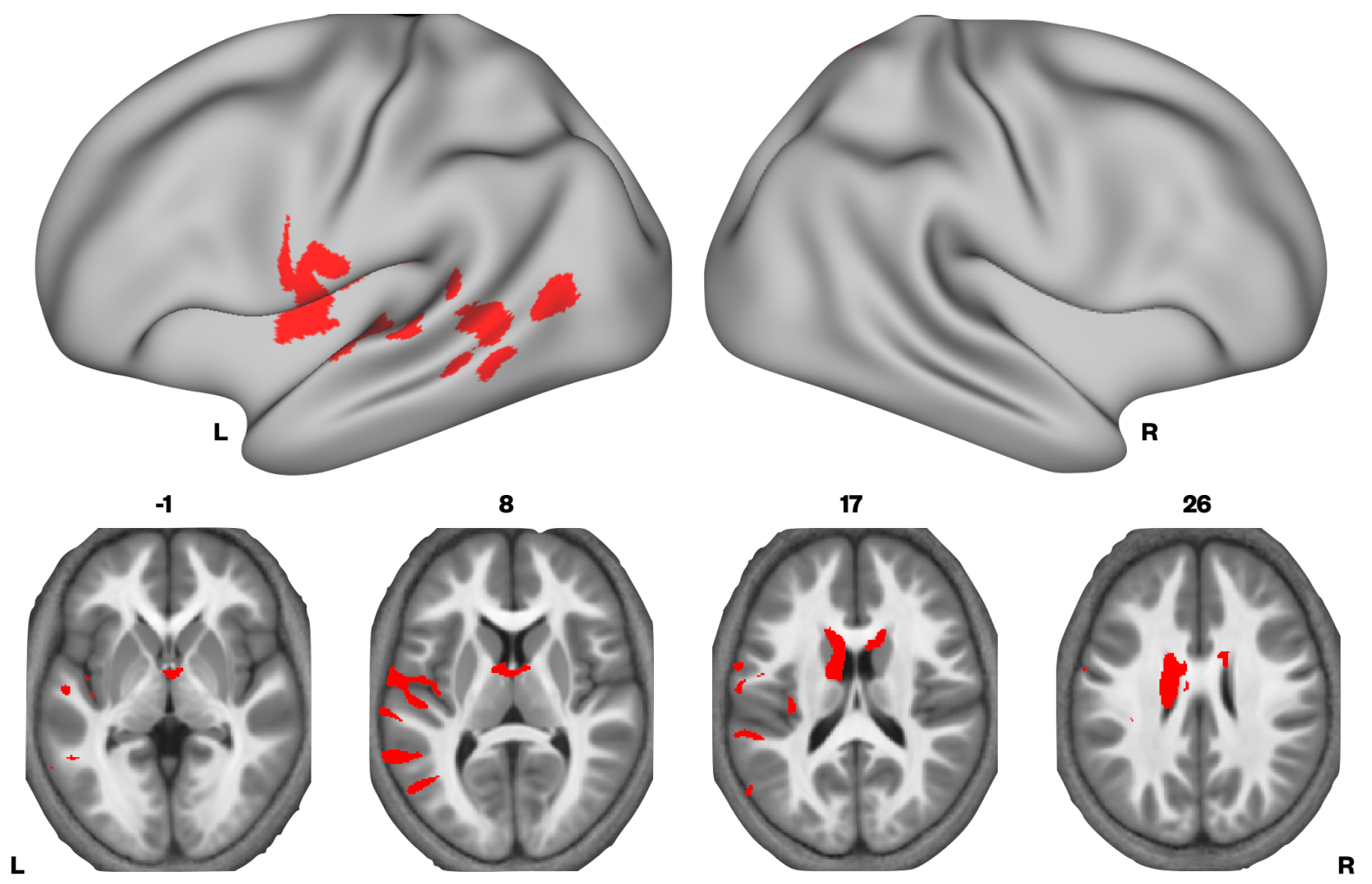

Given our interest in the dorsal striatum in relation to DLD (see introduction), we extracted MTsat values for each participant from the caudate nuclei bilaterally (the region where we observed TD>DLD differences). Using a hemispheric mask, we separated this cluster into left and right components. We then assessed if group differences in MTsat values in the left and right caudate nuclei could be accounted for by age or total intracranial volume. Age and total intracranial volume were not significant predictors of MTsat values in the caudate nuclei clusters, see Supplementary Figure 2.

\section{Whole-brain correlation analysis of neural microstructure with language proficiency}

We have previously found continuous measures of language proficiency to be more sensitive to neural differences than diagnostic categories (Krishnan et al., 2020). Using a continuous measure of language ability also gives us the opportunity to include those children who did not meet our criteria for DLD, but had a history of speech and language problems (HSL). We constructed language and memory factor scores from our neuropsychological battery (see Krishnan et al., 2020), and assessed if these were predictors of our three parameter values in 
this larger set of children. Given the strong correlation between the language and memory factors $(r=0.7, p<.001)$, we entered these predictors separately into our statistical models. We found that language proficiency was strongly correlated with MTsat values focally in the left caudate nucleus (Figure 4). In the R1 maps, poorer language proficiency was once again associated with widespread reduction over the lateral convexities of the frontal and parietal lobes bilaterally (as seen before in the TD > DLD differences, which were slightly more right lateralised), the medial frontal cortex including SMA and extending to paracentral lobule, and left posterior temporal cortex extending from the superior temporal plane to posterior inferior temporal cortex. Subcortically, there were differences in R1 in the dorsal striatum and thalamus bilaterally, and in anterior portions of the medial temporal lobe. These differences were similar to those seen in the TD > DLD contrast. R1 values in a more focal but overlapping set of regions (perisylvian cortex including the inferior frontal gyrus, insula, superior temporal gyrus, extending to the anterior temporal pole, bilaterally, but more extensive on the left, and the dorsal striatum bilaterally) were associated with poor memory proficiency, suggesting that these cognitive differences were reflecting the globally lowered values of R1 in children with DLD. There were no significant relationships between R2* values in grey matter and language or memory proficiency.

\section{TD vs DLD group differences in voxel-based morphometry}

Finally, to assess if the parametric differences reported here were also observed in standard morphometric analysis, we performed a VBM analysis comparing regional amounts of grey matter in TD vs. DLD using T1 scans from the same participants (note these were collected using an MPRAGE T1-weighted sequence, and were not the T1-weighted scan from the MPM protocol). We did not observe any group differences that survived our statistical threshold $(\mathrm{p}<0.05)$, and indeed no group differences were observed at a lower statistical threshold $\mathrm{p}<0.2$. Thus, our parametric differences cannot be explain by morphometric differences in the amount of tissue. 
bioRxiv preprint doi: https://doi.org/10.1101/2021.10.18.464793; this version posted October 20, 2021. The copyright holder for this preprint (which was not certified by peer review) is the author/funder, who has granted bioRxiv a license to display the preprint in perpetuity. It is made available under aCC-BY-NC 4.0 International license.

Figure 4. Correlation between language proficiency and MTsat values in the left caudate nucleus

18

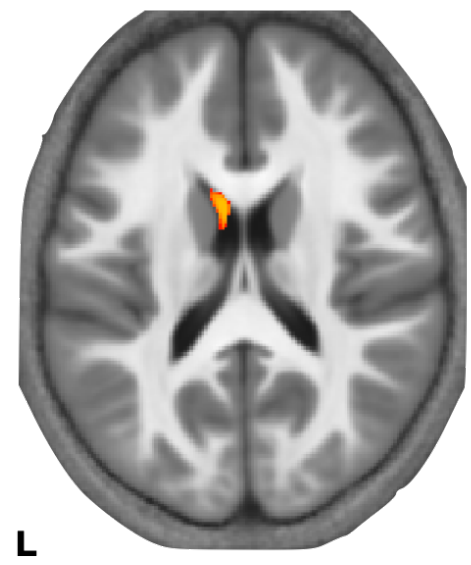

22

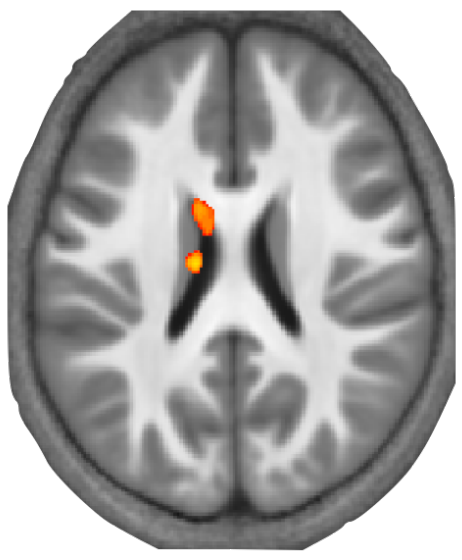

26

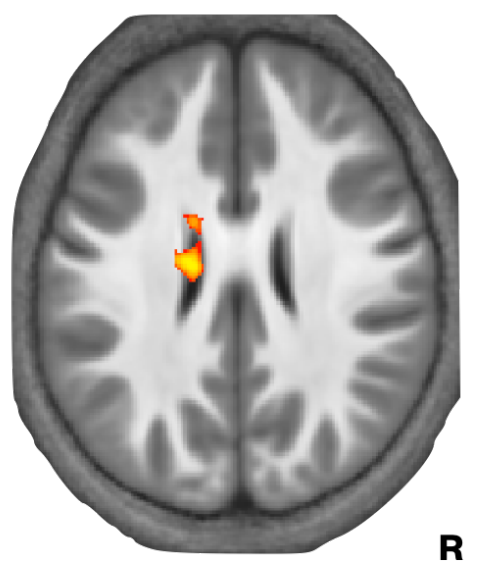

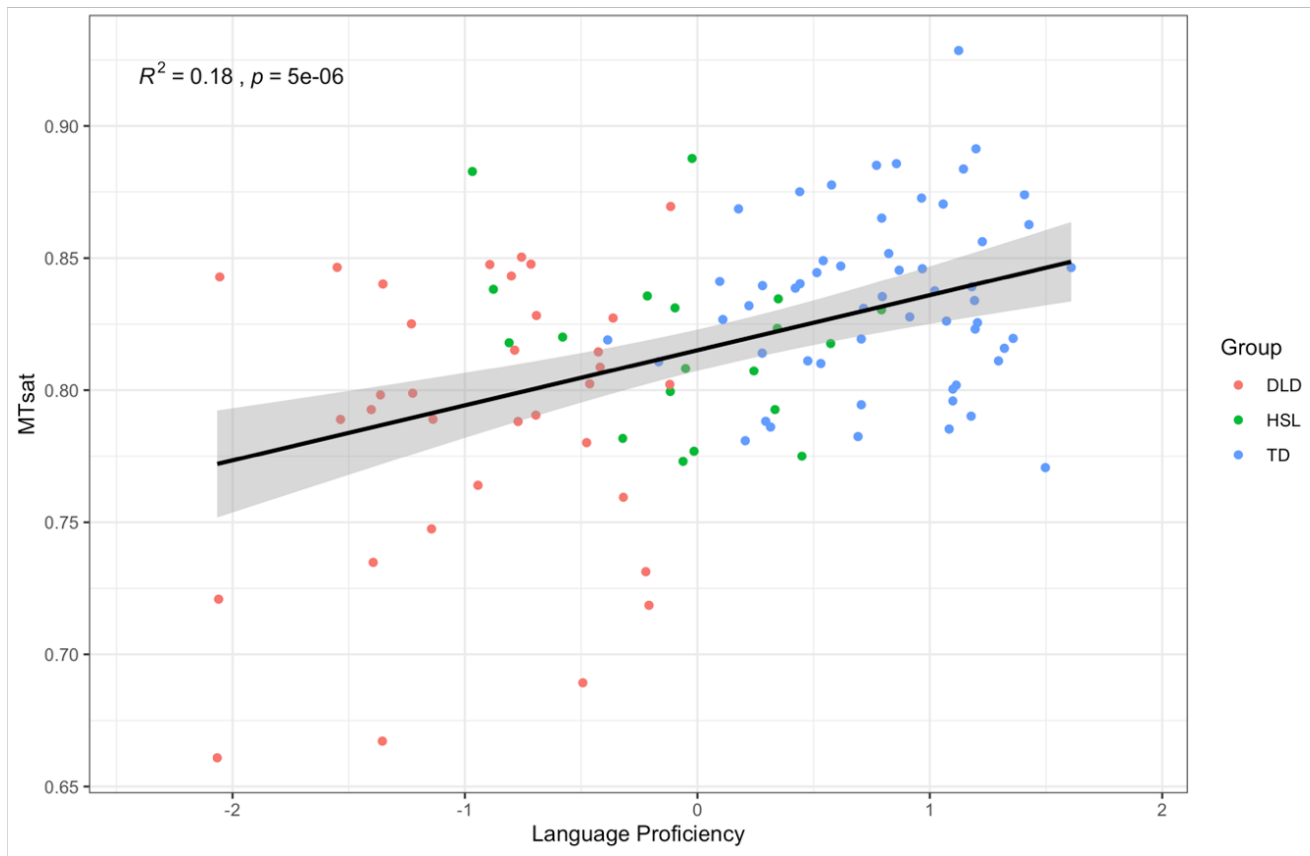

\section{Discussion}

In this study, we used semiquantitative structural MRI to provide a detailed account of the neural differences in children with developmental language disorder (DLD), taking advantage of the sensitivity of this protocol to understand changes in neural microstructure. We found reduced MTsat and R1 values in the caudate nuclei in children with DLD. We also observed reduced MTsat values in the left inferior frontal gyrus. This offers empirical confirmation of 
our prediction that cortico-striato-thalamo-cortical loops involved in learning are affected in DLD.

Importantly, these results allow us to understand the cellular mechanisms driving this change. MTsat and R1 are considered in vivo markers of macromolecular content, and within the brain, these measures are particularly sensitive to myelin content in grey matter. Although most of the brain's myelin is found in the white matter where it sheaths the long axons travelling in white matter fibre tracts, it can also be measured in grey matter where it myelinates axons or parts of axons in the cortex and in subcortical structures like the thalamus and basal ganglia (Nieuwenhuys, 2013). Indeed, myelin is a strong contributor to MR signal in these regions (Glasser \& Essen, 2011; Sereno et al., 2013; Shafee et al., 2015). Given myelin's role in enabling fast and reliable communication in the brain, reduced myelin content may explain why children with DLD struggle with speech and language processing. Below, we discuss these findings, contextualising why myelin may be altered in these specific regions in DLD.

\section{Interpretation of MPM scans}

Myelin is known to increase throughout the brain during adolescence (Paquola et al., 2019; Whitaker et al., 2016), and has been linked to improved behavioural performance (Kwon et al., 2020). Previous morphometric studies have typically drawn inferences about myelin from cortical thinning, but shrinkage of grey matter does not allow us to distinguish if $\mathrm{T} 1$ change is because of shorter T1 times associated with reduced synaptic density, or an increase in the proportion of myelinated neurons (Paus, 2005). More recently, MTsat and R1 measures have been used as an in vivo marker for myelin. These have been validated as markers of myelin through post mortem imaging, as well as histological studies of patients with multiple sclerosis (Weiskopf et al., 2021). Importantly, these maps agree with histological maps showing greater myelin in primary motor and sensory cortex (Carey et al., 2017; Dick et al., 2012; Sereno et al., 2013; Whitaker et al., 2016), as can be seen in our maps as well (Supplementary Figure 2). It is therefore unsurprising to find some convergence over MTsat and R1. R2* is also considered a measure of myelin, but the $\mathrm{T} 2 *$ contrast is particularly sensitive to iron, especially within the basal ganglia. Despite R2* differences being noted in adults with speech problems in the putamen and cortical speech motor network (developmental stuttering, Cler et al., 2021), and observed links between verbal memory and R2* in the ventral striatum (Steiger et al., 
2016), we did not see any evidence for R2* differences anywhere in the brain when comparing our TD and DLD groups.

Our findings also strongly suggest that there is an advantage to using multi-parameter mapping to probe microstructure relative to standard T1 measures. While previous studies reported differences using morphometric measures, we did not observe evidence for morphometric differences in our sample. As others have argued, multi-parameter mapping is sensitive to microstructural differences that would not be detected by a standard voxel-based morphometry (VBM) analysis, as VBM is sensitive to differences in regional amounts of grey matter based on T1-weighting rather than quantitative measurements (Lorio et al., 2014, 2016). Using MPMs allows us to more closely interrogate the histological processes involved in neural changes.

\section{Group differences in the striatum}

Our findings clearly indicate that there are microstructural abnormalities in the caudate nuclei bilaterally in children with DLD, and more broadly, that reduced myelin in the left caudate nucleus is associated with lower language proficiency. The convergence across MT and R1 differences in the caudate nuclei strongly suggest these are linked to abnormal levels of myelin.

The dorsal striatum has been implicated in learning through practice, particularly habit formation (Skipper et al., 2020; Yin \& Knowlton, 2006). A number of groups, including us, have hypothesised that the striatum is a brain region where we might expect to see neural differences in children with DLD (Krishnan et al., 2016; Ullman et al., 2020; Ullman \& Pierpont, 2005). Our hypothesis was driven by the idea that children with DLD showed deficits in sequential procedural tasks, which rely on loops through the striatum (Krishnan et al., 2016). In recent years, some doubt has been cast on the integrity of these behavioural measures for probing sequential learning (West et al., 2018, 2021). Here, using a direct neural measure, we do see evidence for structural differences in the striatum, particularly in the caudate nuclei. The functional consequences of these structural changes might be to make certain aspects of learning, such as the learning of stimulus-response mappings, more challenging. For instance, a reduction in myelin might make sequential learning less efficient. This could explain why children with DLD face difficulties in complex language tasks, such as nonword repetition, where extracting and producing sequential responses are important. However, any speculation 
about the functional impact of these changes needs careful empirical investigation in future studies.

Our findings are generally consistent with previous smaller-scale work linking volumetric differences in the caudate nucleus to language learning difficulties (Badcock et al., 2012; Watkins et al., 2002). They may also offer some resolution to previous work using VBM, where such differences were not observed (Pigdon et al., 2019). Indeed, our own VBM analysis did not reveal any differences in morphometry. However, and perhaps speaking to a slightly different interpretation of our findings, the striatal differences we find are in the body of the caudate nucleus, rather than the head. The head of the caudate nucleus receives input from prefrontal cortex. DTI studies suggest the body of the caudate nucleus gets its input from prefrontal cortex and supplementary motor regions (Lehéricy et al., 2004). Functional connectivity studies indicate that the body of the caudate nucleus also receives projections from temporal association cortex (Choi et al., 2012). Given our findings of differences in the body rather than the head of the caudate nuclei, further studies examining individual differences of microstructure and relevant behavioural tasks (e.g., auditory processing and learning, or processing of rhythm) are warranted.

Although some previous work suggested that striatal differences were normalised by adolescence in children with DLD (Soriano-Mas et al., 2009), our analyses do not show any evidence of group differences being modulated by age (Supplementary Figure 2). Using the MPM protocols may give us a more sensitive marker of change to differences in DLD. In these analyses, it is perhaps surprising that we did not see age-related change in the striatum within the time frame we sampled. Longitudinal work using MTsat has demonstrated that the striatum continues to mature through adolescence, from 14 to 24 years of age (NSPN Consortium et al., 2019). However, it may be that the time frame we sampled was too short, or that within-subject longitudinal studies, which are more sensitive to changes over time, are needed.

\section{Differences beyond the striatum}

We also observed MTsat changes indicative of myelin-related differences in the posterior part of the inferior frontal gyrus, ventral sensorimotor cortex, insula, planum temporale, lateral Heschl's gyrus, and the superior temporal sulcus, all in the left hemisphere. Regions such as the inferior frontal cortex and posterior superior temporal cortex are considered core parts of 
the speech and language network (Ayyash et al., 2021; Fedorenko et al., 2011; Hickok \& Poeppel, 2007; Rauschecker \& Scott, 2009), and ventral sensorimotor cortex and insula are regions that are clearly implicated in speech motor control (Bouchard et al., 2013; Carey et al., 2017; Dronkers, 1996; Wise et al., 1999). Our findings here of abnormal microstructure in these regions are somewhat consistent with those from previous studies in DLD that report structural differences in perisylvian regions (Badcock et al., 2012; Gauger et al., 1997; Jancke et al., 2007; Plante, 1991; Preis et al., 1998) with two notable differences - one, that these differences did not emerge in a VBM analysis, and two, that children with DLD always had lower values that those of children who were typically developing, indicative of slower maturation or abnormal developmental trajectories. Such differences in these regions therefore may be correlates of either auditory or motor inefficiency or both that have been observed in some children with DLD (Halliday et al., 2017; Hill, 2001; McArthur \& Bishop, 2004).

While the TD $>$ DLD differences observed in the MTsat appeared left-lateralised and focal, differences in the R1 map were widespread, observed in both hemispheres and quite symmetrical. Interpreting the differences in these findings offers a paradox. On the face of it, left-lateralised myelin reduction in brain regions known to contribute to speech and language processing seems very plausible, as this would be entirely consistent with the behavioural profile of DLD. This would fit with a popular theoretical view, i.e., that the left hemisphere is uniquely privileged to support language (Vargha-Khadem et al., 1985). However, this does not fit with the developmental literature on children with early brain lesions. Children with perinatal focal brain lesions, even those encompassing the entire left hemisphere, have fairly good language skills, and they typically perform better than children with DLD on language tasks (Asaridou et al., 2020; Bates \& Dick, 2002; Thal et al., 1991). Right hemisphere homologues of language areas are able to support language reorganisation when early damage is sustained (Bates \& Dick, 2002; Newport et al., 2017). This has led to theoretical speculation that abnormalities might need to be bilateral in order for children to develop DLD. In this vein, the widespread differences seen in the R1 maps, or the bilateral abnormalities observed in the caudate nuclei, might point to why organisation of language is not maximally efficient. One possibility is that MTsat mainly indexes myelin differences, whereas R1 could be sensitive to other microstructural features such as iron and neuronal fibres (Edwards et al., 2018). This might suggest that the myelin differences observed in R1, that are not observed in MTsat, indicate further sources of neural difference in DLD. Further work is consequently needed to 
understand what the divergent $\mathrm{TD}>\mathrm{DLD}$ differences across the R1 and MTsat maps might reflect.

It is also notable that many of the MTsat and R1 differences we observe are in primary motor and sensory areas, or closely adjacent areas. Myelin is greatest in primary sensory (due to thalamo-cortical projections) and motor areas (due to the large axons of cortico-spinal projections) and therefore peaks at earlier stages of development (Natu et al., 2019; Paquola et al., 2019; Whitaker et al., 2016). As seen from our average parameter maps (Supplementary Figure 1), we see the expected strong myelination in these regions, and we may therefore have stronger signal in these regions of the brain to evaluate group differences. In contrast, a relative lack of differences in association areas may be a true finding or may reflect reduced sensitivity to measure this change because there is less myelin content there. For instance, in our maps, we do not see very strong myelination in some areas, such as the inferior frontal gyrus, which might limit the ability to see group differences. In typically developing teenagers, myelogenesis is highest in association areas (Whitaker et al., 2016). Longitudinal studies are therefore necessary to evaluate whether differences in myelin persist in the same areas in children with DLD, i.e., regionally specific changes, or whether differences in myelin would be seen in association areas at later stages of development.

We previously hypothesised that the microstructure of grey and white matter in the medial temporal lobe would be relatively normal in children with DLD (Krishnan et al., 2016). However, we observed differences in R1 values in these regions. This also fits with the emerging picture that children with DLD can struggle with aspects of learning thought to depend on the medial temporal lobe, e.g. so-called declarative memory tasks such as list learning (Bishop \& Hsu, 2015; Earle \& Ullman, 2021; Jackson et al., 2020; McGregor et al., 2017).

\section{Limitations and future directions}

The differences we see here are observed at a group level. In other words, lower MT or R1 values in the caudate nuclei are not observed in every child with DLD. In future studies, we are keen to use structural connectivity analyses, as they will allow us to understand how differences across a network of brain areas may make children susceptible to DLD. It is also not clear whether the neural changes we observe are the cause of DLD, or a consequence of having a language disorder. Longitudinal studies where children are followed over time are the 
best way to shed light on this issue. A pertinent issue when considering longitudinal studies is the amount of data we were able to retain (between approximately 65-80\%). We were concerned that systematic biases might affect retention, with children with severe language problems being more likely to be excluded. Consequently, in our sample, we assessed if there were any differences between children whose scans were excluded, relative to those we retained for our analysis. We found that children with DLD whose scans were excluded were younger than those who were selected, but they were not more severely affected in terms of their language learning (Supplementary Table 5). However, it is worth noting our analysis does not account for the children who we recruited but were unable to scan. Dropout and data quality are factors to consider if scanning younger children with this protocol. Finally, the relationship between structure and function is complex. We need to understand how these structural differences might affect specific aspects of function. For instance, we did not observe differences in dorsal striatal activity for a simple language task of verb generation in the same group of children (Krishnan et al., 2020). This may signal the task was not sensitive to differences, or alternately, that we need to tap different aspects of language processing.

\section{Summary and conclusions}

Understanding the neural basis of DLD is particularly challenging given the developmental nature of the disorder, as well as the lack of appropriate animal models for understanding language. Novel semiquantitative MPM protocols allow us an unparalleled in vivo method to investigate microstructural neural changes in these children. Our findings using this protocol suggest that the caudate nucleus, as well as regions in the wider speech and language network, show alterations in myelin in children with DLD. These findings strongly point to a role for the striatum in the development of DLD. This role is likely to be in the learning of habits and sequences, but future work is necessary to test this hypothesis given the anatomical localisation in our study. Additionally, myelin patterns can change over development, and myelination can be observed after successful training. In next steps, it is important to assess whether these differences in myelin persist over development in DLD, and if they can be targeted through training using behavioural interventions. 


\section{Methods}

\section{Ethics}

This study was approved by the Medical Sciences Interdivisional Research Ethics Committee at the University of Oxford (R55835/RE002). Before enrolling participants in the study, we obtained written informed consent from parents/guardians, and written assent from children.

\section{Participants}

As part of the Oxford BOLD study, we recruited and tested 175 children between the ages of 10 and 15 years. All children had to meet certain inclusion/exclusion criteria; specifically, they had to have: (i) normal hearing (defined as passing audiometric screening at $25 \mathrm{~dB}$ at $500 \mathrm{~Hz}$, $1000 \mathrm{~Hz}$, and $2000 \mathrm{~Hz}$, in the better ear); (ii) a nonverbal IQ > 70 (assessed using the WISCIV Matrix Reasoning and Block Design Tests - Wechsler, 2004); and (iii) have grown up in the UK speaking English. Children were excluded if they had another neurodevelopmental disorder such as autism or attention-deficit hyperactivity disorder, or history of neurological disorder. Participants who met inclusionary/exclusionary criteria were categorised as having DLD if they presented with a history of language problems and scored at least 1 SD below the normative mean on two or more standardised tests of language ability. Children were categorised as HSL if they presented with a history of speech and language problems but did not meet criteria for DLD. Those who were categorised as TD had no history of speech and language problems. If these children scored $1 \mathrm{SD}$ or more below the mean on more than one standardised test score of language ability, they were excluded from the TD group. Of the 175 children we recruited, a total of 162 children completed both behavioural testing and MRI scans and met our inclusionary criteria (77 typically developing children, 58 children with DLD, and 27 who had a history of speech and language difficulties (HSL) but did not meet our criteria for DLD at time of testing), for further details, see (Krishnan et al., 2020). From this sample, we acquired MPM data in 72 TD children, 52 children with DLD, and in 24 children with HSL.

\section{Data acquisition}

MR data were collected with a 3T Siemens Prisma scanner with a 32-channel head coil. Participants wore noise-cancelling headphones (Optoacoustics OptoActive II Active Noise Cancelling Headphones). Foam padding was placed around the head for comfort and to restrict movement; the headphones were held in place with inflatable pads. 
Whole brain images at an isotropic resolution of $1 \mathrm{~mm}$ were obtained using an MPM quantitative imaging protocol (Lutti et al., 2014; Weiskopf et al., 2013). This protocol consisted of the acquisition of three multi-echo gradient acquisitions with proton density (PD), T1, or MT weighting. Each acquisition had a TR of $25 \mathrm{~ms}$, field of view $=256 \times 224 \times 176 \mathrm{~mm}^{3}$, readout bandwidth $488 \mathrm{~Hz} /$ pixel, and slab rotation of $30^{\circ}$. Flip angle for MT and PD weighted acquisitions was $6^{\circ}$, and $21^{\circ}$ for T1 weighted acquisitions. MT weighting was achieved by using a Gaussian RF pulse $2 \mathrm{kHz}$ off resonance with $4 \mathrm{~ms}$ duration and a nominal flip angle of $220^{\circ}$ prior to excitation. To speed up data acquisition, a GRAPPA acceleration factor of 2 was applied, with 40 references lines in each phase encoding direction. Eight echoes were acquired for the T1 and PD weighted contrasts, and six echoes were acquired for the MT contrast. Each sequence took approximately 5 minutes to acquire. In addition, data to calculate a radiofrequency $(\mathrm{RF})$ transmit field map was acquired at the start of the session, using a 3D echo-planar imaging spin-echo/stimulated echo method (Lutti et al. 2010, 2012; $\mathrm{FOV}=256 \times 192 \times 192 \mathrm{~mm}^{3}$, matrix $=64 \times 64 \times 48 \mathrm{~mm}^{3}, \mathrm{TE}=39.06$, mixing time $=33.8 \mathrm{~ms}$, $\mathrm{TR}=500 \mathrm{~ms}$, nominal $\alpha$ varying from $115^{\circ}$ to $65^{\circ}$ in steps of $5^{\circ}$, acquisition time $4 \mathrm{~min} 24 \mathrm{~s}$ ). In total, the MPM protocol took approximately 20 minutes to acquire.

We did collect other imaging data as part of the Oxford BOLD study, including fMRI data (Krishnan et al., 2020). Notably, we also obtained a T1-weighted MPRAGE scan (magnetization prepared low angle spoiled gradient echo, TR 1900ms, TE $3.97 \mathrm{~ms}$, flip angle $8^{\circ}$, field of view $208 \times 256 \times 256 \mathrm{~mm}$ ) with $1 \mathrm{~mm}$ in-plane resolution and $1 \mathrm{~mm}$ slice thickness in this set of scans.

\section{Procedure}

MPM data were collected at the end of the scanning session. The session also included two task fMRI scans, a resting state scan, and a diffusion weighted scan; these data are not reported here. During the MPM scans, participants were given the option of either closing their eyes or watching an animated film; nearly all participants chose the film.

Participants also completed a comprehensive neuropsychological battery outside of the scanner, focusing on their linguistic and cognitive abilities (see Krishnan et al., 2020 for further details). In brief, language ability was assessed using five tests, assessing aspects of expressive and receptive grammar, narrative, and vocabulary. Specifically, grammatical comprehension was assessed using the Test for Reception of Grammar - 2 or its electronic counterpart (TROG- 
E, Bishop, 2005). Expressive grammar was evaluated using the Recalling Sentences subtest of the Clinical Evaluation of Language Fundamentals $-4^{\text {th }}$ Edition (CELF-4; Semel et al., 2004). Children's narrative production and comprehension was assessed using the Expression, Reception and Recall of Narrative Instrument (ERNNI; Bishop, 2004). Receptive and expressive vocabulary were assessed using the Receptive One-Word Picture Vocabulary Test - 4th Edition ( ROWPVT-4; Martin and Brownell, 2011b) and Expressive One-Word Picture Vocabulary Test - 4th Edition (EOWPVT-4; Martin and Brownell, 2011a) respectively. In addition to the language measures, children also completed the phonological decoding and sight word reading efficiency subtests of the Test Of Word Reading Efficiency (TOWRE; Torgesen et al., 1999); the block design, matrix reasoning, and coding subtests of the Wechsler Intelligence Scale for Children - $4^{\text {th }}$ Edition (WISC-IV; Wechsler, 2004), the forward and backward digit span subtests, as well as the word lists subtest, from the Children's Memory Scale (CMS; Cohen, 1997), a nonword repetition test (Norbury et al., 2016), and the oromotor sequences sub-test of the NEuroPSYchology (NEPSY) test battery (Korkman et al., 1998).

\section{Data pre-processing}

Data were processed using the hMRI toolbox within SPM12 (Balteau et al., 2018; Tabelow et al., 2019). The default toolbox settings were used. This processing results in the MT saturation, R1 and R2* maps, which index different aspects of tissue microstructure. Briefly, R1 (1/T1) maps were estimated from the PD and $\mathrm{T} 1$ weighted images using the process described in Weiskopf et al. (2013), extended by using correction for radio-frequency transmit field inhomogeneities and imperfect spoiling. Regression of the log signal from the signal decay over echoes across all three MPM contrasts was used to calculate a map of R2* $(=1 / \mathrm{T} 2 *)$ (Weiskopf et al., 2013). RF transmit field maps were calculated from the 3D EPI acquisition and corrected for off-resonance effects as described in (Lutti et al., 2012). The semiquantitative MT saturation parameter (MTsat) calculated is relatively robust against differences in relaxation times and RF transmit and receive field inhomogeneities, and small residual higher dependencies are further corrected for within the toolbox.

Using quality assessment metrics obtained from the toolbox, we removed images where the SD R2* (a measure of image degradation) was greater than 3 times the interquartile range (IQR) from the group mean. Data from 9 children with DLD and 2 TD children were excluded on this basis. We also removed scans where interscan movement exceeded $2 \mathrm{~mm}$. Data from a 
further 4 children with DLD, 5 TD children, and 1 child with HSL were excluded on this basis. We then conducted a visual inspection of the R1, MTsat, and R2* maps, and excluded data from a further 5 children with DLD, 4 children with HSL, and 9 TD children where image artifacts were observed. We retained data from 56 TD children, 34 children with DLD, and 19 children with HSL. This equates to data retention of $77.78 \%$ in the TD population, $65.38 \%$ in the DLD population, and $79.17 \%$ in the HSL population.

Using pipelines implemented in the hMRI toolbox, MTsat maps from each participant were further segmented into grey and white matter probability maps. These grey and white matter maps were used to create a DARTEL template. Each participants' MTsat, R1 and R2* maps were registered to this DARTEL template and were then normalised to a standard MNI template. A tissue weighted smoothing kernel of 6-mm full-width-at-half-maximum was applied using the voxel-based quantification approach (Draganski et al., 2011)., which aims to preserve quantitative values for interpretation.

\section{Data analyses}

We analysed group differences in MTsat, R1 and R2* maps using FSL's randomise tool using 5000 permutations. For assessing statistical differences across groups, we employed thresholdfree cluster enhancement, setting $\mathrm{p}<0.05$ as our threshold. Data from ROIs of interest were extracted using $f_{\text {slstats, }}$ and further analyses were carried out using R (R Core Team, 2020).

For the behavioural data, we constructed factor scores for use in continuous analyses to minimise the number of comparisons in statistical tests. These factors were based on analysis of the whole cohort of children who contributed behavioural data to our study and therefore includes data from children who did not complete the MPM scans and children in whom we excluded MPM data as describe above. The measures from the language and memory tests described above were entered into a pre-registered factor analysis to identify the best weighted combination of measures to give a language factor score, and a memory factor score. The approach we adopted to factor analysis was E-CFA (Brown, 2006), implemented in lavaan (Rosseel, 2012) in the R programming language (R Core Team, 2020). E-CFA is a hybrid exploratory-confirmatory approach to factor analysis where a model is specified with an 'anchor' measure or two anchor measures. As anchor measures, we used the list learning standard score from the CMS for the memory factor, and expressive vocabulary for the 
language factor. We planned to compare this two-factor model to a single factor model accounting for language proficiency alone. However, our two preregistered models were not a good fit to the data. Consequently, as detailed in Krishnan et al. (2020), we accounted for strong correlations between expressive and receptive vocabulary scores, as well as the two narrative production measures in modified models, and found the modified two-factor model to be a better fit to the data than the modified single-factor model. We consequently derived language and memory proficiency scores using this modified two-factor model.

\section{Data availability}

The data that support the findings of this study are openly available on the OSF (https://osf.io/d93gq/?view_only=c48c989c574a49cba6eba1c413f185bb). Statistical maps can also be viewed on Neurovault (https://neurovault.org/collections/DUGBDBPH/)

\section{Acknowledgements}

We thank all of our participants and their families, without whom this work would not be possible. We would also like to acknowledge the many individuals and organisations that helped us with recruitment (https://boldstudy.wordpress.com/acknowledgements/). We especially thank Professor Dorothy Bishop for her support, insight, and discussions throughout OxBOLD. We also thank members of the Wellcome Centre for Integrative Neuroimaging, especially the MRI team at the Oxford Centre for Human Brain Activity: Sebastian Rieger, Juliet Semple, Nicky Aikin, Nicola Filippini, Eniko Zsoldos, and Emily Hinson. We are grateful to Professor Fred Dick, as well as members of the Oxford Speech and Brain Group for helpful discussions and support.

\section{Funding}

The Oxford Brain Organisation in Language Development or OxBOLD study was funded by the Medical Research Council MR/P024149/1 and supported by the NIHR Oxford Health Biomedical Research Centre. The Wellcome Centre for Integrative Neuroimaging is supported by core funding from the Wellcome Trust (203139/Z/16/Z). 


\section{Competing interests}

The authors report no competing interests.

\section{References}

Allen, M., Glen, J. C., Müllensiefen, D., Schwarzkopf, D. S., Fardo, F., Frank, D., Callaghan, M. F., \& Rees, G. (2017). Metacognitive ability correlates with hippocampal and prefrontal microstructure. NeuroImage, 149, 415-423. https://doi.org/10.1016/j.neuroimage.2017.02.008

Argyropoulos, G. P. D., Watkins, K. E., Belton-Pagnamenta, E., Liégeois, F., Saleem, K. S., Mishkin, M., \& Vargha-Khadem, F. (2019). Neocerebellar Crus I Abnormalities Associated with a Speech and Language Disorder Due to a Mutation in FOXP2. The Cerebellum, 18(3), 309-319. https://doi.org/10.1007/s12311-018-0989-3

Asaridou, S. S., Demir-Lira, Ö. E., Goldin-Meadow, S., Levine, S. C., \& Small, S. L. (2020). Language development and brain reorganization in a child born without the left hemisphere. Cortex, 127, 290-312. https://doi.org/10.1016/j.cortex.2020.02.006 Ayyash, D., Malik-Moraleda, S., Gallée, J., Affourtit, J., Hoffman, M., Mineroff, Z., Jouravlev, O., \& Fedorenko, E. (2021). The universal language network: A crosslinguistic investigation spanning 45 languages and 11 language families [Preprint]. Neuroscience. https://doi.org/10.1101/2021.07.28.454040

Badcock, N. A., Bishop, D. V. M., Hardiman, M. J., Barry, J. G., \& Watkins, K. E. (2012). Co-localisation of abnormal brain structure and function in specific language impairment. Brain and Language, 120(3), 310-320. https://doi.org/10.1016/j.bandl.2011.10.006

Balteau, E., Tabelow, K., Ashburner, J., Callaghan, M. F., Draganski, B., Helms, G., Kherif, F., Leutritz, T., Lutti, A., Phillips, C., Reimer, E., Ruthotto, L., Seif, M., Weiskopf, N., Ziegler, G., \& Mohammadi, S. (2018). HMRI - A toolbox for using quantitative MRI in neuroscience and clinical research. 37.

Bates, E., \& Dick, F. (2002). Language, gesture, and the developing brain. Developmental Psychobiology, 40(3), 293-310. https://doi.org/10.1002/dev.10034

Belton, E., Salmond, C. H., Watkins, K. E., Vargha-Khadem, F., \& Gadian, D. G. (2003). Bilateral brain abnormalities associated with dominantly inherited verbal and orofacial dyspraxia. Human Brain Mapping, 18(3), 194-200.

https://doi.org/10.1002/hbm.10093 
Bishop, D. V. M. (2004). Expression, Reception and Recall of Narrative Instrument: ERRNI. Harcourt assessment.

Bishop, D. V. M. (2005). Test for Reception of Grammar, Version 2-Electronic (TROG-E). (2nd ed.). The Psychological Corporation.

Bishop, D. V. M., \& Hsu, H. J. (2015). The declarative system in children with specific language impairment: A comparison of meaningful and meaningless auditory-visual paired associate learning. BMC Psychology, 3(1), 3. https://doi.org/10.1186/s40359015-0062-7

Bouchard, K. E., Mesgarani, N., Johnson, K., \& Chang, E. F. (2013). Functional organization of human sensorimotor cortex for speech articulation. Nature, 495(7441), 327-332. https://doi.org/10.1038/nature11911

Brown, T. A. (2006). Confirmatory factor analysis for applied research. Guilford Press. Callaghan, M. F., Freund, P., Draganski, B., Anderson, E., Cappelletti, M., Chowdhury, R., Diedrichsen, J., FitzGerald, T. H. B., Smittenaar, P., Helms, G., Lutti, A., \& Weiskopf, N. (2014). Widespread age-related differences in the human brain microstructure revealed by quantitative magnetic resonance imaging. Neurobiology of Aging, 35(8), 1862-1872. https://doi.org/10.1016/j.neurobiolaging.2014.02.008

Carey, D., Caprini, F., Allen, M., Lutti, A., Weiskopf, N., Rees, G., Callaghan, M. F., \& Dick, F. (2018). Quantitative MRI provides markers of intra-, inter-regional, and agerelated differences in young adult cortical microstructure. NeuroImage, 182, 429-440. https://doi.org/10.1016/j.neuroimage.2017.11.066

Carey, D., Krishnan, S., Callaghan, M. F., Sereno, M. I., \& Dick, F. (2017). Functional and Quantitative MRI Mapping of Somatomotor Representations of Human Supralaryngeal Vocal Tract. Cerebral Cortex, 27(1), 265-278. https://doi.org/10.1093/cercor/bhw393

Choi, E. Y., Yeo, B. T. T., \& Buckner, R. L. (2012). The organization of the human striatum estimated by intrinsic functional connectivity. Journal of Neurophysiology, 108(8), 2242-2263. https://doi.org/10.1152/jn.00270.2012

Clark, I. A., Callaghan, M. F., Weiskopf, N., \& Maguire, E. A. (2020). Hippocampal grey matter tissue microstructure does not explain individual differences in hippocampaldependent task performance. BioRxiv, 2020.08.18.255992. https://doi.org/10.1101/2020.08.18.255992 
Cler, G., Krishnan, S., Papp, D., Wiltshire, C., Chesters, J., \& Watkins, K. E. (2021). Elevated iron concentration in putamen and cortical speech motor network in developmental stuttering. OSF Preprints. https://doi.org/10.31219/osf.io/ypq8s

Cohen, M. (1997). Children's memory scale. Psychological Corporation.

Conti-Ramsden, G., Durkin, K., Toseeb, U., Botting, N., \& Pickles, A. (2018). Education and employment outcomes of young adults with a history of developmental language disorder. International Journal of Language \& Communication Disorders, 53(2), 237-255. https://doi.org/10.1111/1460-6984.12338

Dick, F., Taylor Tierney, A., Lutti, A., Josephs, O., Sereno, M. I., \& Weiskopf, N. (2012). In Vivo Functional and Myeloarchitectonic Mapping of Human Primary Auditory Areas. Journal of Neuroscience, 32(46), 16095-16105. https://doi.org/10.1523/JNEUROSCI.1712-12.2012

Draganski, B., Ashburner, J., Hutton, C., Kherif, F., Frackowiak, R. S. J., Helms, G., \& Weiskopf, N. (2011). Regional specificity of MRI contrast parameter changes in normal ageing revealed by voxel-based quantification (VBQ). NeuroImage, 55(4), 1423-1434. https://doi.org/10.1016/j.neuroimage.2011.01.052

Dronkers, N. (1996). A new brain region for coordinating speech articulation. Nature, 384, $159-161$.

Earle, F. S., \& Ullman, M. T. (2021). Deficits of Learning in Procedural Memory and Consolidation in Declarative Memory in Adults With Developmental Language Disorder. Journal of Speech, Language, and Hearing Research, 64(2), 531-541. https://doi.org/10.1044/2020_JSLHR-20-00292

Edwards, L. J., Kirilina, E., Mohammadi, S., \& Weiskopf, N. (2018). Microstructural imaging of human neocortex in vivo. NeuroImage, 182, 184-206. https://doi.org/10.1016/j.neuroimage.2018.02.055

Fedorenko, E., Behr, M. K., \& Kanwisher, N. (2011). Functional specificity for high-level linguistic processing in the human brain. Proceedings of the National Academy of Sciences, 108(39), 16428-16433. https://doi.org/10.1073/pnas.1112937108

Freund, P., Weiskopf, N., Ashburner, J., Wolf, K., Sutter, R., Altmann, D. R., Friston, K., Thompson, A., \& Curt, A. (2013). MRI investigation of the sensorimotor cortex and the corticospinal tract after acute spinal cord injury: A prospective longitudinal study. The Lancet Neurology, 12(9), 873-881. https://doi.org/10.1016/S14744422(13)70146-7 
Gauger, L. M., Lombardino, L. J., \& Leonard, C. M. (1997). Brain morphology in children with specific language impairment. Journal of Speech, Language, and Hearing Research; Rockville, 40(6), 1272-1284.

https://search.proquest.com/docview/232349104/abstract/BD6AB18BF9924CCBPQ/ 1

Girbau-Massana, D., Garcia-Marti, G., Marti-Bonmati, L., \& Schwartz, R. G. (2014). Graywhite matter and cerebrospinal fluid volume differences in children with Specific Language Impairment and/or Reading Disability. Neuropsychologia, 56, 90-100. https://doi.org/10.1016/j.neuropsychologia.2014.01.004

Glasser, M. F., \& Essen, D. C. V. (2011). Mapping Human Cortical Areas In Vivo Based on Myelin Content as Revealed by T1- and T2-Weighted MRI. Journal of Neuroscience, 31(32), 11597-11616. https://doi.org/10.1523/JNEUROSCI.2180-11.2011

Graybiel, A. M., \& Grafton, S. T. (2015). The Striatum: Where Skills and Habits Meet. Cold Spring Harbor Perspectives in Biology, 7(8), a021691. https://doi.org/10.1101/cshperspect.a021691

Halliday, L. F., Tuomainen, O., \& Rosen, S. (2017). Auditory processing deficits are sometimes necessary and sometimes sufficient for language difficulties in children: Evidence from mild to moderate sensorineural hearing loss. Cognition, 166, 139-151. https://doi.org/10.1016/j.cognition.2017.04.014

Herbert, M. R., Ziegler, D. A., Makris, N., Bakardjiev, A., Hodgson, J., Adrien, K. T., Kennedy, D. N., Filipek, P. A., \& Caviness, V. S. (2003). Larger brain and white matter volumes in children with developmental language disorder. Developmental Science, 6(4), F11-F22. https://doi.org/10.1111/1467-7687.00291

Hickok, G., \& Poeppel, D. (2007). The cortical organization of speech processing. Nature Reviews Neuroscience, 8(5), 393-402. https://doi.org/10.1038/nrn2113

Hill, E. L. (2001). Non-specific nature of specific language impairment: A review of the literature with regard to concomitant motor impairments. International Journal of Language \& Communication Disorders, 36(2), 149-171. https://doi.org/10.1080/13682820010019874

Hsu, H. J., \& Bishop, D. V. M. (2014). Sequence-specific procedural learning deficits in children with specific language impairment. Developmental Science, 17(3), 352-365. https://doi.org/10.1111/desc.12125 
Huttenlocher, P. R. (1979). Synaptic density in human frontal cortex-Developmental changes and effects of aging. Brain Research, 163(2), 195-205. https://doi.org/10.1016/0006-8993(79)90349-4

Jackson, E., Leit, ão S., Claessen, M., \& Boyes, M. (2020). Working, Declarative, and Procedural Memory in Children With Developmental Language Disorder. Journal of Speech, Language, and Hearing Research, 63(12), 4162-4178. https://doi.org/10.1044/2020_JSLHR-20-00135

Jancke, L., Siegenthaler, T., Preis, S., \& Steinmetz, H. (2007). Decreased white-matter density in a left-sided fronto-temporal network in children with developmental language disorder: Evidence for anatomical anomalies in a motor-language network. Brain and Language, 102(1), 91-98. https://doi.org/10.1016/j.bandl.2006.08.003

Jernigan, T. L., Hesselink, J. R., Sowell, E., \& Tallal, P. A. (1991). Cerebral structure on magnetic resonance imaging in language- and learning-impaired children. Archives of Neurology, 48(5), 539-545.

Korkman, M., Kirk, U., \& Kemp, S. L. (1998). NEPSY: Neuropsychological assessment of Children. Psychological Corporation.

Krishnan, S., Asaridou, S. S., Cler, G. J., Smith, H. J., Willis, H. E., Healy, M. P., Thompson, P. A., Bishop, D. V. M., \& Watkins, K. E. (2020). Functional organisation for verb generation in children with developmental language disorder. NeuroImage, 117599. https://doi.org/10.1016/j.neuroimage.2020.117599

Krishnan, S., Watkins, K. E., \& Bishop, D. V. M. (2016). Neurobiological Basis of Language Learning Difficulties. Trends in Cognitive Sciences, 20(9), 701-714. https://doi.org/10.1016/j.tics.2016.06.012

Kurth, F., Luders, E., Pigdon, L., Conti-Ramsden, G., Reilly, S., \& Morgan, A. T. (2018).

Altered gray matter volumes in language-associated regions in children with developmental language disorder and speech sound disorder. Developmental Psychobiology, 60(7), 814-824. https://doi.org/10.1002/dev.21762

Kwon, D., Pfefferbaum, A., Sullivan, E. V., \& Pohl, K. M. (2020). Regional growth trajectories of cortical myelination in adolescents and young adults: Longitudinal validation and functional correlates. Brain Imaging and Behavior, 14(1), 242-266. https://doi.org/10.1007/s11682-018-9980-3

Langkammer, C., Krebs, N., Goessler, W., Scheurer, E., Ebner, F., Yen, K., Fazekas, F., \& Ropele, S. (2010). Quantitative MR Imaging of Brain Iron: A Postmortem Validation Study. Radiology, 257(2), 455-462. https://doi.org/10.1148/radiol.10100495 
Lee, J. C., Dick, A. S., \& Tomblin, J. B. (2020). Altered brain structures in the dorsal and ventral language pathways in individuals with and without developmental language disorder (DLD). Brain Imaging and Behavior. https://doi.org/10.1007/s11682-01900209-1

Lee, J. C., Nopoulos, P. C., \& Bruce Tomblin, J. (2013). Abnormal subcortical components of the corticostriatal system in young adults with DLI: A combined structural MRI and DTI study. Neuropsychologia, 51(11), 2154-2161. https://doi.org/10.1016/j.neuropsychologia.2013.07.011

Lehéricy, S., Ducros, M., Van De Moortele, P.-F., Francois, C., Thivard, L., Poupon, C., Swindale, N., Ugurbil, K., \& Kim, D.-S. (2004). Diffusion tensor fiber tracking shows distinct corticostriatal circuits in humans: DTI Corticostriatal Fibers. Annals of Neurology, 55(4), 522-529. https://doi.org/10.1002/ana.20030

Leutritz, T., Seif, M., Helms, G., Samson, R. S., Curt, A., Freund, P., \& Weiskopf, N. (2020). Multiparameter mapping of relaxation ( $\mathrm{R} 1, \mathrm{R} 2 *)$, proton density and magnetization transfer saturation at $3 \mathrm{~T}$ : A multicenter dual-vendor reproducibility and repeatability study. Human Brain Mapping, 41(15), 4232-4247. https://doi.org/10.1002/hbm.25122

Lorio, S., Kherif, F., Ruef, A., Melie-Garcia, L., Frackowiak, R., Ashburner, J., Helms, G., Lutti, A., \& Draganski, B. (2016). Neurobiological origin of spurious brain morphological changes: A quantitative MRI study. Human Brain Mapping, 37(5), 1801-1815. https://doi.org/10.1002/hbm.23137

Lorio, S., Lutti, A., Kherif, F., Ruef, A., Dukart, J., Chowdhury, R., Frackowiak, R. S., Ashburner, J., Helms, G., Weiskopf, N., \& Draganski, B. (2014). Disentangling in vivo the effects of iron content and atrophy on the ageing human brain. NeuroImage, 103, 280-289. https://doi.org/10.1016/j.neuroimage.2014.09.044

Lum, J. A. G., Conti-Ramsden, G., Morgan, A. T., \& Ullman, M. T. (2014). Procedural learning deficits in specific language impairment (SLI): A meta-analysis of serial reaction time task performance. Cortex, 51, 1-10. https://doi.org/10.1016/j.cortex.2013.10.011

Lutti, A., Dick, F., Sereno, M. I., \& Weiskopf, N. (2014). Using high-resolution quantitative mapping of R1 as an index of cortical myelination. NeuroImage, 93, 176-188. https://doi.org/10.1016/j.neuroimage.2013.06.005

Manara, R., Ponticorvo, S., Tartaglione, I., Femina, G., Elefante, A., Russo, C., Carafa, P. A., Cirillo, M., Casale, M., Ciancio, A., Di Concilio, R., De Michele, E., Weiskopf, N., 
Di Salle, F., Perrotta, S., \& Esposito, F. (2019). Brain iron content in systemic iron overload: A beta-thalassemia quantitative MRI study. NeuroImage: Clinical, 24, 102058. https://doi.org/10.1016/j.nicl.2019.102058

Martin, N. A., \& Brownell, R. (2011a). Expressive One-Word Picture Vocabulary Test-4 (EOWPVT-4). (4th ed.). Academic Therapy Publications.

Martin, N. A., \& Brownell, R. (2011b). Receptive One-Word Picture Vocabulary Test-4 (ROWPVT-4). (4th ed.). Academic Therapy Publications.

Mayes, A. K., Reilly, S., \& Morgan, A. T. (2015). Neural correlates of childhood language disorder: A systematic review. Developmental Medicine \& Child Neurology, 57(8), 706-717. https://doi.org/10.1111/dmcn.12714

McArthur, G. M., \& Bishop, D. V. M. (2004). Which People with Specific Language Impairment have Auditory Processing Deficits? Cognitive Neuropsychology, 21(1), 79-94. https://doi.org/10.1080/02643290342000087

McGregor, K. K., Gordon, K., Eden, N., Arbisi, -Kelm Tim, \& Oleson, J. (2017). Encoding Deficits Impede Word Learning and Memory in Adults With Developmental Language Disorders. Journal of Speech, Language, and Hearing Research, 60(10), 2891-2905. https://doi.org/10.1044/2017_JSLHR-L-17-0031

Natu, V. S., Gomez, J., Barnett, M., Jeska, B., Kirilina, E., Jaeger, C., Zhen, Z., Cox, S., Weiner, K. S., Weiskopf, N., \& Grill-Spector, K. (2019). Apparent thinning of human visual cortex during childhood is associated with myelination. Proceedings of the National Academy of Sciences, 116(41), 20750-20759. https://doi.org/10.1073/pnas.1904931116

Newport, E. L., Landau, B., Seydell-Greenwald, A., Turkeltaub, P. E., Chambers, C. E., Dromerick, A. W., Carpenter, J., Berl, M. M., \& Gaillard, W. D. (2017). Revisiting Lenneberg's Hypotheses About Early Developmental Plasticity: Language Organization After Left-Hemisphere Perinatal Stroke. Biolinguistics, 11, 407-422. https://www.ncbi.nlm.nih.gov/pmc/articles/PMC6291004/

Nieuwenhuys, R. (2013). The myeloarchitectonic studies on the human cerebral cortex of the Vogt-Vogt school, and their significance for the interpretation of functional neuroimaging data. Brain Structure and Function, 218(2), 303-352.

https://doi.org/10.1007/s00429-012-0460-z

Norbury, C. F., Gooch, D., Wray, C., Baird, G., Charman, T., Simonoff, E., Vamvakas, G., \& Pickles, A. (2016). The impact of nonverbal ability on prevalence and clinical 
presentation of language disorder: Evidence from a population study. Journal of Child Psychology and Psychiatry, 57(11), 1247-1257. https://doi.org/10.1111/jcpp.12573

NSPN Consortium, Ziegler, G., Hauser, T. U., Moutoussis, M., Bullmore, E. T., Goodyer, I. M., Fonagy, P., Jones, P. B., Lindenberger, U., \& Dolan, R. J. (2019). Compulsivity and impulsivity traits linked to attenuated developmental frontostriatal myelination trajectories. Nature Neuroscience, 22(6), 992-999. https://doi.org/10.1038/s41593019-0394-3

Paquola, C., Bethlehem, R. A., Seidlitz, J., Wagstyl, K., Romero-Garcia, R., Whitaker, K. J., Vos de Wael, R., Williams, G. B., NSPN Consortium, Vértes, P. E., Margulies, D. S., Bernhardt, B., \& Bullmore, E. T. (2019). Shifts in myeloarchitecture characterise adolescent development of cortical gradients. ELife, 8, e50482. https://doi.org/10.7554/eLife.50482

Paus, T. (2005). Mapping brain maturation and cognitive development during adolescence. Trends in Cognitive Sciences, 9(2), 60-68. https://doi.org/10.1016/j.tics.2004.12.008

Pigdon, L., Willmott, C., Reilly, S., Conti-Ramsden, G., Gaser, C., Connelly, A., \& Morgan, A. T. (2019). Grey matter volume in developmental speech and language disorder. Brain Structure \& Function, 224(9), 3387-3398. https://doi.org/10.1007/s00429-01901978-7

Plante, E. (1991). MRI findings in boys with specific language impairment. Brain and Language, 41(1), 52-66. https://doi.org/10.1016/0093-934X(91)90110-M

Preis, S., JÄncke, L., Schittler, P., Huang, Y., \& Steinmetz, H. (1998). Normal intrasylvian anatomical asymmetry in children with developmental language disorder. Neuropsychologia, 36(9), 849-855. https://doi.org/10.1016/S0028-3932(98)00033-5

R Core Team. (2020). R: A language and environment for statistical computing. $\mathrm{R}$ Foundation for Statistical Computing. https://www.R-project.org/.

Rauschecker, A. M., Pringle, A., \& Watkins, K. E. (2008). Changes in neural activity associated with learning to articulate novel auditory pseudowords by covert repetition. Human Brain Mapping, 29(11), 1231-1242. https://doi.org/10.1002/hbm.20460

Rauschecker, J. P., \& Scott, S. K. (2009). Maps and streams in the auditory cortex:

Nonhuman primates illuminate human speech processing. Nature Neuroscience, 12(6), 718-724. https://doi.org/10.1038/nn.2331

Rosseel, Y. (2012). lavaan: An R package for structural equation modeling and more Version 0.5-12 (BETA). 37. 
Schmierer, K., Scaravilli, F., Altmann, D. R., Barker, G. J., \& Miller, D. H. (2004).

Magnetization transfer ratio and myelin in postmortem multiple sclerosis brain. Annals of Neurology, 56(3), 407-415. https://doi.org/10.1002/ana.20202

Semel, E. M., Wiig, E. H., \& Secord, W. (2004). Clinical Evaluation of Language Fundamentals. (4th ed.). PsychCorp.

Sereno, M. I., Lutti, A., Weiskopf, N., \& Dick, F. (2013). Mapping the Human Cortical Surface by Combining Quantitative T1 with Retinotopy $\uparrow$. Cerebral Cortex, 23(9), 2261-2268. https://doi.org/10.1093/cercor/bhs213

Shafee, R., Buckner, R. L., \& Fischl, B. (2015). Gray matter myelination of 1555 human brains using partial volume corrected MRI images. NeuroImage, 105, 473-485. https://doi.org/10.1016/j.neuroimage.2014.10.054

Simmonds, A. J., Leech, R., Iverson, P., \& Wise, R. J. S. (2014). The response of the anterior striatum during adult human vocal learning. Journal of Neurophysiology, 112(4), 792-801. https://doi.org/10.1152/jn.00901.2013

Skipper, J. I., Aliko, S., Brown, S., Jo, Y. J., Lo, S., Molimpakis, E., \& Lametti, D. R. (2020). Reorganization of the neurobiology of language after sentence overlearning [Preprint]. Neuroscience. https://doi.org/10.1101/2020.09.11.293167

Soriano-Mas, C., Pujol, J., Ortiz, H., Deus, J., López-Sala, A., \& Sans, A. (2009). Agerelated brain structural alterations in children with specific language impairment. Human Brain Mapping, 30(5), 1626-1636. https://doi.org/10.1002/hbm.20620

Steiger, T. K., Weiskopf, N., \& Bunzeck, N. (2016). Iron Level and Myelin Content in the Ventral Striatum Predict Memory Performance in the Aging Brain. Journal of Neuroscience, 36(12), 3552-3558. https://doi.org/10.1523/JNEUROSCI.361715.2016

Tabelow, K., Balteau, E., Ashburner, J., Callaghan, M. F., Draganski, B., Helms, G., Kherif, F., Leutritz, T., Lutti, A., Phillips, C., Reimer, E., Ruthotto, L., Seif, M., Weiskopf, N., Ziegler, G., \& Mohammadi, S. (2019). HMRI - A toolbox for quantitative MRI in neuroscience and clinical research. NeuroImage, 194, 191-210. https://doi.org/10.1016/j.neuroimage.2019.01.029

Thal, D. J., Marchman, V., Stiles, J., Aram, D., Trauner, D., Nass, R., \& Bates, E. (1991). Early lexical development in children with focal brain injury. Brain and Language, 40(4), 491-527. https://doi.org/10.1016/0093-934X(91)90145-Q

Torgesen, J. K., Rashotte, C. E., \& Wagner, R. K. (1999). TOWRE: Test of word reading efficiency. Pro-ed. 
Ullman, M. T., Earle, F. S., Walenski, M., \& Janacsek, K. (2020). The Neurocognition of Developmental Disorders of Language. Annual Review of Psychology, 71(1), 389417. https://doi.org/10.1146/annurev-psych-122216-011555

Ullman, M. T., \& Pierpont, E. I. (2005). Specific Language Impairment is not Specific to Language: The Procedural Deficit Hypothesis. Cortex, 41(3), 399-433. https://doi.org/10.1016/S0010-9452(08)70276-4

Vargha-Khadem, F., O’Gorman, A. M., \& Watters, G. V. (1985). Aphasia and handedness in relation to hemispheric side, age at injury and severity of cerebral lesion during childhood. Brain, 108(3), 677-696. https://doi.org/10.1093/brain/108.3.677

Watkins, K. E., Vargha-Khadem, F., Ashburner, J., Passingham, R. E., Connelly, A., Friston, K. J., Frackowiak, R. S. J., Mishkin, M., \& Gadian, D. G. (2002). MRI analysis of an inherited speech and language disorder: Structural brain abnormalities. Brain, 125(3), 465-478. https://doi.org/10.1093/brain/awf057

Wechsler, D. (2004). WISC-IV: Wechsler Intelligence Scale for Children: Technical and Interpretive Manual. Psychological Corporation.

Weiskopf, N., Edwards, L. J., Helms, G., Mohammadi, S., \& Kirilina, E. (2021). Quantitative magnetic resonance imaging of brain anatomy and in vivo histology. Nature Reviews Physics, 1-19. https://doi.org/10.1038/s42254-021-00326-1

Weiskopf, N., Suckling, J., Williams, G., Correia, M. M., Inkster, B., Tait, R., Ooi, C., Bullmore, E. T., \& Lutti, A. (2013). Quantitative multi-parameter mapping of R1, PD*, MT, and R2* at 3T: A multi-center validation. Frontiers in Neuroscience, 7. https://doi.org/10.3389/fnins.2013.00095

West, G., Melby-Lervåg, M., \& Hulme, C. (2021). Is a procedural learning deficit a causal risk factor for developmental language disorder or dyslexia? A meta-analytic review. Developmental Psychology, 57(5), 749-770. https://doi.org/10.1037/dev0001172

West, G., Vadillo, M. A., Shanks, D. R., \& Hulme, C. (2018). The procedural learning deficit hypothesis of language learning disorders: We see some problems. Developmental Science, 21(2), e12552. https://doi.org/10.1111/desc.12552

Whitaker, K. J., Vértes, P. E., Romero-Garcia, R., Váša, F., Moutoussis, M., Prabhu, G., Weiskopf, N., Callaghan, M. F., Wagstyl, K., Rittman, T., Tait, R., Ooi, C., Suckling, J., Inkster, B., Fonagy, P., Dolan, R. J., Jones, P. B., Goodyer, I. M., the NSPN Consortium, \& Bullmore, E. T. (2016). Adolescence is associated with genomically patterned consolidation of the hubs of the human brain connectome. Proceedings of 
the National Academy of Sciences, 113(32), 9105-9110.

https://doi.org/10.1073/pnas.1601745113

Wise, R., Greene, J., Büchel, C., \& Scott, S. (1999). Brain regions involved in articulation.

The Lancet, 353(9158), 1057-1061. https://doi.org/10.1016/S0140-6736(98)07491-1

Yin, H. H., \& Knowlton, B. J. (2006). The role of the basal ganglia in habit formation.

Nature Reviews Neuroscience, 7(6), 464-476. https://doi.org/10.1038/nrn1919 